\title{
Reversores de los nuevos anticoagulantes
}

\section{Reversal agents of new anticoagulants}

\section{Juan de Jesús Montenegro-Aldana ${ }^{a, b, *}$, Diego Andrés Rodríguez-Guerrero ${ }^{a, b, c}$, Luis Carlos Sáenz-Morales ${ }^{a, c}$ y José Fernando López ${ }^{\mathrm{d}}$}

\author{
a Centro Internacional de Arritmias, Instituto de Cardiología, Fundación Cardioinfantil, Bogotá, Colombia \\ b Clínica Universitaria Colombia, Bogotá, Colombia \\ c Facultad de Medicina, Universidad de La Sabana, Bogotá, Colombia \\ 'Electrofisiología, Clínica del Country, Bogotá, Colombia
}

Recibido el 3 de octubre de 2016; aceptado el 6 de octubre de 2016

Disponible en Internet el 4 de noviembre de 2016

\section{Introducción}

La fibrilación auricular y su presencia en los pacientes afectados han sufrido un notorio incremento a través de los años. La incidencia aumentó alrededor de un 13\% en las últimas dos décadas, con un riesgo de desarrollarla a lo largo de la vida del $25 \%$. La principal complicación son los eventos tromboembólicos, los cuales conllevan incapacidad y cambios de la calidad de vida de los pacientes que la padecen. Se calcula que uno de cada cinco ataques cerebro-vasculares se atribuye a fibrilación auricular y que el riesgo de muerte a causa de un ataque cerebro-vascular asociado a fibrilación auricular, es el doble comparado con el no asociado a esta $^{1}$. Es así que, para la prevención y el tratamiento de estos eventos, se han desarrollado anticoagulantes directos orales (DOAC) como alternativa a los anticoagulantes (ACO) antes disponibles dependientes de la vitamina $\mathrm{K}$ (-warfarinaúnico disponible comercialmente en Colombia). Este artículo se propone revisar los datos clínicos disponibles de los agentes hemostáticos antagonistas de los DOAC.

\footnotetext{
* Autor para correspondencia.

Correo electrónico: jmontald@hotmail.com (J.J. Montenegro-Aldana).
}

Los anticoagulantes antagonistas de la vitamina $\mathrm{K}$ (coumarínicos) tienen desafíos en la estabilidad y el mantenimiento de su acción farmacológica, incremento del riesgo de sangrados extra- e intracraneales, además de su vida media prolongada entre 20 y 60 horas, características que ponen límites a su uso en la práctica clínica. Los diferentes estudios realizados con los DOAC desarrollados en prevención de eventos tromboembólicos en fibrilación auricular, muestran no inferioridad respecto a los antagonistas de la vitamina K. Aunque se haya establecido un aumento del riesgo de hemorragias con el uso de los ACO y el perfil de eficacia y de seguridad esté a favor de los DOAC, se tiene conocimiento de la ausencia de un agente específico para la reversión de su actividad anticoagulante. Si bien en algunas situaciones clínicas, teniendo en cuenta la corta vida media, la sola suspensión de los DOAC y medidas generales que pueden ser suficientes, en otros contextos clínicos de sangrados amenazantes para la vida, se puede requerir una pronta reversión de su actividad anticoagulante. En ciertas situaciones como sobredosis, sangrados masivos, necesidad de una pronta restauración de la hemostasia por compromiso hemodinámico o en periodos preoperatorios, pueden necesitarse estos antagonistas de los DOAC. En otros casos clínicos donde no hay compromiso hemodinámico, la suspensión de los anticoagulantes, un adecuado soporte y el control del sitio de sangrado son la base del manejo. 
El uso de concentrados de complejo de protrombina (PCC) y factor VII activado, ha tenido cierto grado de eficacia ${ }^{2}$. La hemodiálisis puede remover hasta el $60 \%$ de dabigatrán circulante. El uso de carbón activado también puede ser útil en la reducción de la absorción del dabigatrán aplicado en las dos primeras horas de la ingesta de este y en las primeras seis horas de la ingesta de rivaroxabán y apixabán ${ }^{3}$.

\section{Métodos}

Se efectuó una estrategia de búsqueda sistemática de la literatura para identificar los estudios potenciales relevantes de los últimos diez años en Medline, Embase, Clinical Key y en el Registro Cochrane, utilizando la interfaz de OVID SP. Se obtuvieron 73 artículos relacionados con el tema de manejo del sangrado en pacientes en tratamiento con ACO.

\section{¿Cuándo reversar los anticoagulantes directos orales?}

Los pacientes que se podrían beneficiar de los nuevos agentes antagonistas de la ACO se encuentran en situaciones clínicas donde ha habido sobredosis accidental o intencional, sangrado amenazante para la vida, hemorragias del sistema nervioso central o que serán sometidos a cirugía de urgencia donde no es posible la suspensión de los DOAC por 12 - 24 horas; sin embargo, no existen estudios que evalúen la evolución clínica que demuestren una disminución de la morbi-mortalidad cuando se utilizan estos antagonistas de los DOAC. Las recomendaciones del manejo del sangrado en pacientes en tratamiento con estos anticoagulantes, se aplican con base en la experiencia y el reflejo de la opinión de expertos.

El primer paso es definir qué es un sangrado crítico amenazante para la vida o una hemorragia masiva; esta última es arbitraria, aunque cualquiera de las definiciones que se usan en la literatura puede tener el valor de iniciar la logística de manejo del sangrado. Entre las definiciones más comunes de sangrado masivo e incontrolable están la pérdida de sangre que requiere la reposición total del volumen sanguíneo en menos de 24 horas o reposiciones del 50\% en tres horas. Si se puede contabilizar el sangrado, se consideran hemorragias masivas aquellas pérdidas superiores a $150 \mathrm{ml} / \mathrm{min}$ por más de $10 \mathrm{~min}$ o sangrados superiores a $1,5 \mathrm{ml} / \mathrm{kg} / \mathrm{min}$ durante más de $20 \mathrm{~min}$, o hemorragia mayor que precisa transfusión de 4 concentrados de hematíes en una hora ${ }^{4}$. También se deben considerar las causas más frecuentes de sangrado amenazantes para la vida y que requieren pronta valoración y manejo: politraumatismos, cirugía cardiovascular, hemorragia postparto, sangrado digestivo y cirugía hepatobiliar y del sistema nervioso central.

\section{Antídotos o reversores de los anticoagulantes directos orales}

Los antídotos para los DOAC están en pleno desarrollo. En la actualidad se dispone de tres agentes para la reversión del efecto de estos anticoagulantes:
- Andexanet alfa: también conocido como PRT064445. Es un derivado recombinante modificado del factor $\mathrm{Xa}$ en desarrollo, como agente de reversión directo para todas las pequeñas moléculas inhibidoras del factor Xa (por ejemplo, rivaroxabán, apixabán, edoxabán y betrixabán), para las heparinas de bajo peso molecular (HBPM) y para fondaparinux ${ }^{5}$.

- Ciraparantag: se conoce también como PER977, anteriormente como aripazine, y es una pequeña molécula sintética que se une a los inhibidores del factor Xa: dabigatrán y heparinas ${ }^{6}$.

- Idarucizumab: también conocido como Bl655075, es un anticuerpo monoclonal humanizado de ratón, fragmento de anticuerpo $(F A B)$, que se une a dabigatrán con alta afinidad. En octubre de 2015 este medicamento fue aprobado por la Administración de Alimentos y Medicamentos Estadounidense (Food and Drug Administration, FDA) y posteriormente, el 20 de noviembre de 2015, en Europa por la Agencia Europea de Medicamentos (European Medicines Agency, EMA). Estos agentes han demostrado eficacia en revertir los DOAC y reducir la hemorragia en estudios preclínicos, con un perfil de seguridad favorable. Múltiples estudios que evalúan la eficacia y la seguridad de estos agentes, están en curso en la actualidad.

\section{Andexanet alfa}

Fue desarrollado como una molécula recombinante del factor Xa modificada para usarla como un agente de reversión en pacientes que reciben un inhibidor del factor $\mathrm{Xa}$, y que sufren un episodio de sangrado o que puede requerir cirugía de emergencia. Los estudios en entorno clínico no están en curso y los datos disponibles son ensayos realizados en voluntarios sanos. Andexanet alfa actúa como un señuelo del factor Xa con alta afinidad tanto en su forma directa como indirecta por el factor inhibidor de Xa (DOAC: rivaroxabán, apixabán y edoxabán; HBPM y fondaparinux). Una vez unido el andexanet alfa a los inhibidores del factor $\mathrm{Xa}$, estos son incapaces de unirse al inhibir el factor Xa nativo, lo que permite la restauración de procesos hemostáticos normales ${ }^{5}$.

Andexanet alfa no requiere ningún paso de activación para los factores VIla (de la vía extrínseca) o IXa (de la vía intrínseca). Andexanet alfa es rápido y activamente eliminado (vida media de 30-60 min), no exhibe efectos detectables procoagulantes 0 actividad anticoagulante, tal como se plantea en un ensayo de coagulación al examinar los efectos de rivaroxabán y andexanet alfa en el plasma humano sobre el tiempo de protrombina. Su efecto sobre la reversión de la inhibición de factor $\mathrm{X}$ a es dosis dependiente. A dos minutos de finalizado un bolo intravenoso de andexanet alfa, la actividad anti FXa disminuyó en más del $90 \%$ y se mantuvo durante la infusión. En un periodo de infusión de $2 \mathrm{~h}$, la reversión completa de la inhibición de la generación de trombinas se mantuvo durante la infusión y luego de $2 \mathrm{~h}$ después de terminada, mientras que la actividad anti FXa regresó a niveles similares a los del grupo control. Las dosis que se han utilizado varían de $90-600 \mathrm{mg}$ una vez en bolo, seguido de una infusión continua a $8 \mathrm{mg} / \mathrm{min}$ durante $1 \mathrm{~h}$; han sido bien toleradas y no se han reportado eventos adversos. Hoy existen estudios en fase III en los que se 
evalúan los efectos para la reversión de la anticoagulación con apixabán en voluntarios sanos de edad avanzada ${ }^{7}$.

\section{Idarucizumab}

Es un fragmento de anticuerpo monoclonal con una afinidad por el dabigatrán, aproximadamente 350 veces mayor que la afinidad del dabigratán por la trombina. La unión al dabigratán se realiza tanto en su forma libre como en la unida a la trombina, impidiendo así su adhesión a la trombina y neutralizando el efecto anticoagulante. En experimentos in vitro de plasma humano enriquecido con dabigatrán, la adición de idarucizumab inhibió por completo el efecto anticoagulante. En un modelo animal en murinos con hemorragia intracraneal, el idarucizumab redujo la expansión de hematoma intracraneal, y en casos de hemorragia extensa disminuyó la mortalidad. En ensayos clínicos en fase । y $\|$ realizados en voluntarios sanos, idarucizumab revirtió el efecto anticoagulante de dabigatrán en un lapso de $5 \mathrm{~min}$, de manera dependiente de la dosis; se evaluó mediante el tiempo de trombina diluida, tiempo de trombina, tiempo parcial de protrombina, tiempo de coagulación activado y parámetros de tromboelastografía ${ }^{8}$. La administración de idarucizumab en ausencia de dabigatrán no tuvo efecto sobre los parámetros de coagulación o potencial endógeno de la trombina, lo que sugiere que es poco probable que sea protrombótico. Ha sido probado en dosis crecientes en bolo único de $1 \mathrm{a} 8 \mathrm{~g}$, así como en infusión continua. En estos ensayos, fue bien tolerado y no presentó efectos trombóticos ni se informaron efectos secundarios graves. Basándose en estos resultados, en abril de 2015, la FDA concedió una revisión prioritaria a idarucizumab. En el estudio Reversión de dabigatrán anticoagulante Efecto con Idarucizumab (REVERSA AD), que es un estudio clínico abierto en fase III, de un solo brazo, se ensayó la administración de idarucizumab en pacientes que cursaran con hemorragia grave o que requirieran intervenciones quirúrgicas de emergencia y que estuvieran en tratamiento con dabigatrán. A los pacientes elegibles se les dieron $5 \mathrm{~g}$ de idarucizumab intravenoso en dos bolos de $50 \mathrm{ml}$, cada uno de $2,5 \mathrm{~g}$ de idarucizumab con $15 \mathrm{~min}$ de diferencia. El objetivo principal de valoración fue el porcentaje máximo de reversión del efecto anticoagulante de dabigatrán al final de la infusión de idarucizumab y 4 horas después de la segunda infusión. Se evaluó el tiempo de trombina y el de coagulación de ecarina. En este análisis intermedio, idarucizumab normalizó los resultados de la prueba en $88-98 \%$ de los pacientes en cuestión de minutos. En los pacientes tratados con dabigatrán que se someten a procedimientos de urgencia o cirugías, la administración de idarucizumab se asoció con hemostasia intraoperatoria normal en el $94,3 \%$ de los $\operatorname{casos}^{9}$. Fue bien tolerado, sin efectos secundarios trombóticos o formación de efectos secundarios graves.

\section{Ciparantag}

También conocido como PER977 o aripazine es una pequeña molécula sintética, soluble en agua, molécula catiónica diseñada para unirse a todos los DOAC, tanto al factor lla o al factor Xa como también a las heparinas a través de un enlace no covalente de hidrógeno. Su mecanismo de acción impide la unión de los DOAC a su objetivo endógeno, actuando de esta forma en la reversión del efecto anticoagulante.
El ciparantag tendría el potencial de ser un reversor universal de los diferentes anticoagulantes directos actualmente disponibles. En un estudio experimental con ratones, revirtió el efecto ACO de dabigatrán, rivaroxabán, apixabán y endoxabán. Un ensayo de fase । de 180 voluntarios sanos, ciraparantag administrado a una dosis de 100 a $300 \mathrm{mg}$, revirtió inmediatamente $(10 \mathrm{~min})$ los efectos de anticoagulación exhibidos por la mayor dosis de edoxabán y se mantuvo durante las siguientes $24 \mathrm{~h}$. No se observó evidencia de un efecto protrombótico tal como se determina por los fragmentos de protrombina, dímero $\mathrm{D}$, o factor tisular inhibidor de la vía intrínseca y extrínseca. En la actualidad, hay ensayos clínicos en fase I y ॥ para evaluar la eficacia y seguridad de aripazine y se espera que sus resultados sean anunciados en los próximos uno a dos años ${ }^{10}$.

\section{Conclusiones}

El advenimiento de los DOAC constituye un gran avance en la tromboprofilaxis y el tratamiento de la enfermedad tromboembólica. La facilidad de su uso, la amplia ventana terapéutica y el hecho que no requieren control de la anticoagulación y la necesidad solo de ajustes terapéuticos dependientes de la función renal, se ha traducido en el aumento de la utilización de esta clase de medicamentos. Un obstáculo importante asociado con su uso es el temor del paciente y del médico de presentar sangrados importantes y la necesidad de revertir el efecto anticoagulante.

Aunque la necesidad de una reversión inmediata del efecto anticoagulante es rara, la expansión de la utilización de los DOAC hará más frecuente este efecto indeseable. La llegada de estos "antídotos" reversores del efecto anticoagulante, puede dar mayor tranquilidad en la utilización clínica.

Se esperan estudios clínicos que avalen estas perspectivas de control del sangrado indeseado o cuando se requieran cirugías de urgencia en pacientes que se beneficien del uso de los DOAC. Hasta el momento, el manejo de estos pacientes debe incluir:

1. Suspensión inmediata de los DOAC.

2. Medidas hemodinámicas de apoyo o reprogramación de la cirugía durante $12-24 \mathrm{~h}$ después de la última dosis administrada.

3. Consideración de la utilización de factores hemostáticos PPC.

4. Medidas generales del manejo de cualquier sangrado, incluyendo manejo del sitio comprometido.

\section{Conflicto de intereses}

Los autores declaran no tener ningún conflicto de intereses.

\section{Bibliografía}

1. Camm J, Kirchhof P, Lip GYH, et al. Guías ESC FA 2010. Rev Esp Cardiol. 2010;63:e1-83.

2. Suryanarayan D, Schulman S. Potential antidotes for reversal of old and new oral anticoagulants. Thromb Res. 2014;133 Suppl 2:S158-66. 
3. Dalal J, Bhave A, Chaudhry G, et al. Reversal agents for DOACs: Connecting the dots. Indian Heart J. 2016;68:559-63.

4. Llau JV, Acosta FJ, Escolarc G, et al. Documento multidisciplinar de consenso sobre el manejo de la hemorragia masiva (documento HEMOMAS). Rev Esp Anestesiol Reanim. 2016;63:e1-22.

5. Lu G, De Guzman FR, Hollenback SJ, et al. A specific antidote for reversal of anticoagulat on by director indirect inhibitors factor Xa. Nat Med. 2013;19:446-51.

6. Laulicht B, Bakhru S, Lee C, Baker C, Jiang X, Mathiowitz E, Costin J, Steiner S. Abstract 11395: small molecule antidote for anticoagulants. Circulation. 2012;126 21 Suppl:A11395.

7. Crowther M, Levy GG, Lu G, et al. A phase 2 randomized, double-blind, placebo-controlled trial demonstrating reversal of edoxaban-induced anticoagulation in healthy subjects by andexanet alfa (PRT064445): a universal antidote for factor Xa (fXa) inhibitors. Blood. 2014;124:4269.

8. Glund S, Moschetti V, Norris S, et al. A randomized study in healthy volunteers to investigate the safety, tolerability and pharmacokinetics of idarucizumab, a specific antidote to dabigatran. Thromb Haemost. 2015;113:943-51.

9. Pollack CV Jr, Reilly PA, Eikelboom J, et al. Idarucizumab for dabigatran reversal. N Engl J Med. 2015;373:511-20.

10. Ansell JE, Bakhru SH, Laulicht BE, et al. Use of PER977 to reverse the anticoagulant effect of edoxaban. N Engl J Med. 2014;371:2141-2. 